\title{
Philosophiques
}

\section{Et si l'on ne faisait rien de Carl Schmitt ?}

\section{Olivier Jouanjan}

Volume 39, numéro 2, automne 2012

URI : https://id.erudit.org/iderudit/1013701ar

DOI : https://doi.org/10.7202/1013701ar

Aller au sommaire du numéro

Éditeur(s)

Société de philosophie du Québec

ISSN

0316-2923 (imprimé)

1492-1391 (numérique)

Découvrir la revue

Citer ce document

Jouanjan, O. (2012). Et si l'on ne faisait rien de Carl Schmitt? Philosophiques, 39(2), 475-482. https://doi.org/10.7202/1013701ar d'utilisation que vous pouvez consulter en ligne.

https://apropos.erudit.org/fr/usagers/politique-dutilisation/ 


\title{
Et si l'on ne faisait rien de Carl Schmitt?
}

\author{
OLIVIER JOUANJAN \\ professeur de droit public à l'Université de Strasbourg \\ professeur honoraire à l'Université de Fribourg-en-Brisgau \\ olivier.jouanjan@unistra.fr
}

Jean-François Kervégan pose la question "Que faire de Carl Schmitt?", même si son ouvrage aurait dû préférablement s'intituler: "Que faire avec — et contre - Carl Schmitt ?" puisque la seconde partie de son livre est une «tentative pour "penser avec et contre Carl Schmitt" »1.

Christian Nadeau nous invite à la disputatio, manière ancienne, scolastique mais surtout magnifique de philosopher ou, simplement, de penser nos objets. Je dis «nos» objets parce que Schmitt est aussi pour moi un objet de réflexion et d'interrogation et donc, par là-même, un objet de dispute. Dans les conditions modernes, une disputatio impose que les sujets en controverse parlent à la première personne du singulier. C'est d'ailleurs la façon dont Kervégan nous parle dans sa défense, non pas de Carl Schmitt, mais de sa relation à Carl Schmitt, une relation d'ailleurs évolutive et, de ce point de vue, toujours plus distanciée à l'égard de Schmitt. On me permettra donc d'entrer en dispute avec Jean-François Kervégan, moi aussi à la première personne.

Je dois dire d'emblée que l'objet de la dispute n'est absolument pas dans l'ordre du reproche: reprocher d'avoir lu Carl Schmitt, ni même et surtout, reprocher d'avoir voulu penser quelque chose (je laisse ce quelque chose indéterminé) à partir de (sinon avec) Carl Schmitt. Il faut lire Schmitt et penser Schmitt (laissons ouverte la question d'un penser avec - même si c'est finalement contre - Schmitt). Avec son livre, Kervégan marque sa distance d'avec Schmitt, et la question est seulement de savoir jusqu'à quel point ce regard distancié est encore redevable de quoi que ce soit à Schmitt dans la considération qu'il prend de ses objets.

Je n'ai pas la compétence universelle qui me permettrait de répondre à Jean-François Kervégan sur l'ensemble des problèmes et des questions que soulève son livre. Ce sont des questions profondes. Je m'autorise donc à parler seulement du point de vue qui est le mien, à savoir la science du droit et la théorie du droit. De ce seul point de vue, je voudrais essayer de dire en peu de mots qu'il n'y a pas grand-chose à penser ni avec ni contre Schmitt. Pour ce faire je me servirai d'un auteur évoqué en passant dans le livre de Kervégan, Hermann Heller. Sans doute s'attend-on à lire le nom de Hans Kelsen, l'ennemi théorique apparemment le plus radical de Schmitt, le «normativiste » en lutte contre le « décisionniste » Schmitt. Mais rappeler le débat

1. Kervégan, Que faire de Carl Schmitt?, Gallimard, coll. "Tel », 20I I, p. 76. 
Schmitt/Kelsen, si bien connu, me semblerait manquer une position somme toute plus radicale encore que celle de Kelsen contre Schmitt. Je voudrais donc montrer que Hermann Heller détruit beaucoup plus radicalement encore que Kelsen la posture théorique de Schmitt sur le droit, que Kervégan sent bien, dans son livre, que l'alternative Schmitt/Kelsen réduit, appauvrit et, en fin de compte, émascule le débat théorique sur le droit, mais que, malheureusement, il n'a pas apprécié à sa juste mesure cette voie hellérienne qui nous conduit jusqu'à ce point où l'on peut penser que, du point de vue de la théorie du droit à tout le moins, il n’y a pas grand-chose à faire de Carl Schmitt ${ }^{2}$.

Kervégan cite Heller parmi les juristes de gauche ayant pris intérêt, sous Weimar, à l'œuvre de Carl Schmitt ${ }^{3}$. Cela me paraît trop vite dit. Certes, Heller s'est intéressé à Schmitt, mais comme on peut s'intéresser à son ennemi principal et, plus précisément encore à son ennemi politique principal. En effet, d'une part, ce que l'on pourrait considérer comme théorie du droit chez Schmitt apparaît à Heller ${ }^{4}$ faible et même inconsistant; d'autre part, cette théorie schmittienne étant essentiellement politique, détruire la théorie, c'est en même temps détruire la politique qui la sous-tend, et réciproquement. Mon interprétation est donc que si Heller s'intéresse à Schmitt, c'est moins pour l'intérêt qu'aurait la doctrine juridique de ce dernier qu'à raison de l'influence politique que cette doctrine inconsistante exerce malgré tout sur la jeune génération des juristes de Weimar, surtout à droite, mais aussi, marginalement, à gauche. Le véritable adversaire théorique de Heller s'appelle Hans Kelsen, parce que critiquer et démonter la cohérence logique du normativisme de ce dernier constitue un défi d'une tout autre ampleur que celui qui doit simplement se borner à montrer l'irrationalisme du mythe politique schmittien ${ }^{5}$.

Cette opposition politique (et donc aussi «théorique») frontale Heller/ Schmitt s'expose de la façon la plus claire et décisive à l'occasion du procès Prusse contre Reich, au lendemain du «coup d'État» fomenté le 20 juillet I932 par le chancelier du Reich, Franz von Papen, contre le gouvernement

2. Pour le développement de cette critique hellérienne de Schmitt (et de Kelsen) que je me borne ici à résumer, je me permets de renvoyer à la présentation que j'ai faite de ma traduction d'un texte important de Heller: Hermann Heller/Olivier Jouanjan, La crise de la théorie de l'État, Dalloz, 2012 (avec les références).

3. Kervégan, op. cit., p. 26.

4. Comme à Kelsen: voir la contre-attaque foudroyante que ce dernier mène contre le Schmitt de Le gardien de la constitution dans Qui doit être le gardien de la constitution? (trad. fr. par S. Baume, Paris, Michel Houdiard, 2006) et qui montre toutes les contradictions internes, toute l'inconsistance de la théorie schmittienne.

5. Pour prendre toute la mesure de la «considération» que Heller pouvait porter à Schmitt, on peut rappeler cette notation de Heller dans sa Staatslehre (inachevée du fait de sa mort en exil en novembre I933) selon laquelle il n'était pas injustifié de soumettre "l'activisme inconsistant ami/ennemi de Schmitt» à un «traitement psychanalytique»: Heller, Gesammelte Schriften, $2^{\mathrm{e}}$ éd., Tübingen, Mohr Siebeck, I992, t. 3, p. 3 I 4 . 
prussien social-démocrate d'Otto Braun ${ }^{6}$. Durant le procès qui se déroule devant la Cour constitutionnelle de Weimar (Staatsgerichtshof), Heller explique: "Comme y insiste toujours et à juste titre mon collègue Schmitt — sur ce point je lui donne raison - "politique", et donc aussi politique au sens partisan, ce n'est jamais que l'autre; il va de soi que nous-mêmes, nous sommes parfaitement apolitiques, objectifs et au-dessus des partis ${ }^{7}$.» Par cette petite phrase, Heller ne s'oppose pas seulement à une technique argumentative de Schmitt au procès (il précise: Schmitt y insiste "toujours"), mais il met en évidence la mécanique même de toute la rhétorique juridicopolitique schmittienne de manière, à mon sens, juste et profonde.

Rappelons qu'il avait été procédé à la suspension du gouvernement prussien par une ordonnance de nécessité du président Hindenburg (à l'instigation de Papen) fondée, sans entrer ici dans le détail, sur le célèbre article 48 de la Constitution de Weimar autorisant l'exécutif du Reich à prendre les mesures nécessaires lorsqu'un Land ne remplit pas ses obligations fédérales ou lorsqu'il existe dans un Land une menace grave et immédiate pour la sécurité publique. L'ordonnance du 20 juillet 1932 invoquait d'ailleurs cumulativement ces deux raisons pour justifier la suspension du cabinet prussien. Tandis que la ligne de défense des autres représentants de la Prusse au procès consistait à montrer que les conditions d'une telle ordonnance n'étaient pas réunies en fait (la Prusse n'avait nullement manqué à ses obligations fédérales, il n'existait pas de menace sur la sécurité publique à laquelle la Prusse n'aurait pu faire face), la ligne de défense de Heller était différente: il est notoire que le Cabinet Papen a engagé une nouvelle politique à l'égard de la NSDAP rompant avec l'attitude hostile de Brüning pour donner des gages de bonne volonté à Hitler, une politique de désescalade donc. L'ordonnance de juillet s'inscrit dans le cadre de cette politique. Elle n'a donc pas été prise pour des motifs qui pourraient la justifier au titre de l'art. 48 , mais pour un motif strictement politique, et doit être de ce fait tenue pour inconstitutionnelle. Tel était l'argument principal de Heller, et l'historien peut le trouver aujourd'hui parfaitement justifié.

Or, durant les débats, Schmitt a pour ainsi dire corroboré l'argument de Heller. En effet, dans sa première intervention, Schmitt avait justifié le changement de pratique à l'égard de la NSDAP engagé dès le mois de juin par Papen comme une tentative pour diriger l'État de manière «indépendante et impartiale» et que, de ce point de vue prétendument neutre, il était «juste et objectif» de mettre fin à cette "égalité de traitement» avec le parti

6. Kervégan rappelle cet affrontement procédural, même si, techniquement, Carl Schmitt n'était pas «l'avocat» du Reich mais l'un des représentants défendant le gouvernement central et Heller, pas «l'avocat de la Prusse», mais le représentant de la Diète prussienne. Il ne s'agit que d'une précision juridique technique. Kervégan, op. cit., p. 54.

7. Les débats ont été publiés par des juristes proches du SPD dans: Preußen contra Reich. Stenogrammbericht der Verhandlungen vor dem Staatsgerichtshof in Leipzig vom 10. bis 14. und vom 17. Oktober 1932, Berlin, Dietz Verlag, I933, ici: p. 76. 
communiste, une égalité «insultante pour un mouvement avec lequel des millions d'Allemands ne se contentent pas de sympathiser, mais auquel ils ont donné leurs voix ${ }^{8}$.

Voilà donc, à mon sens, ce que la remarque de Heller met en évidence, qui s'exprime clairement certes dans la conjoncture du coup d'État de Prusse, mais qui traverse l'œuvre de Schmitt depuis au moins le texte de I92 I sur la dictature. Cette intervention constitue, je crois, un tournant fondamental dans la pensée schmittienne ${ }^{9}$ : le "politique ", c'est-à-dire la défense d'un parti, c'est toujours l'autre, car la "politique» de Papen de désescalade envers les nazis doit être dite un acte «juste et objectif » pris par une autorité «indépendante et impartiale». Le réalisme politique de Heller nous dit: qui peut croire à cette légende? Mais il nous dit aussi: qui ne voit que cette légende est en dernier ressort fondée sur le mythe politique de Carl Schmitt?

En effet, cette affaire nous enjoint de réfléchir un peu plus profondément au rôle que la "situation d'exception » joue dans la pensée de Schmitt. Elle est donnée pour la révélatrice de la situation politique par excellence. Elle révèle que le droit tout entier, dont les normes ne peuvent valoir que dans la situation "normale", repose sur une "décision » souveraine et donc sur une décision "politique» fondamentale. Or, ce cas pratique (et le cas est plus parlant d'une théorie juridique que n'importe quel discours abstrait) nous montre précisément comment Schmitt voit la "décision souveraine »: une décision "juste et objective» prise par une instance "indépendante et impartiale». Heller nous dit: c'est donc la décision clairement dépendante de la situation et partiale en faveur de la NSDAP, et contre le parti communiste, que Schmitt nous demande de considérer comme la décision souveraine "indépendante et impartiale»! Le souverain schmittien qui tranche dans la situation hyper-politique, la situation d'exception, s'élèverait donc ainsi jusqu'à une position somme toute apolitique d'indépendance et d'impartialité pour prendre la décision «juste et objective». On a envie de dire: la décision «neutre». Mais le mot «neutre» est un mot banni de la politique juridique schmittienne, c'est le mot favori de l'«État de droit bourgeois». C'est pourtant la position que revendique Schmitt dans le litige hyperpolitique Prusse contre Reich.

Or, ce que précisément nous masque la politique schmittienne (ami/ ennemi), pour trouver son propre fondement mythologique, c'est que, dans la situation politique extrême, la décision ne peut être que "partisane» et, en l'occurrence, donner la prime à la NSDAP par rapport au parti communiste, qui reçoit aussi les voix d'un nombre significatif d'électeurs à l'époque. À ce moment précis, dans la mythologie schmittienne, intervient le «peuple».

8. Preußen contra Reich, op. cit., p. 39. Cette déclaration de l'automne 1932 devrait sans doute faire réfléchir ceux qui tiennent encore à la thèse d'une corruption tardive de la pensée de Schmitt et seulement conditionnée par la prise de pouvoir de janvier 1933.

9. La dictature (I92I), trad. fr. par M. Köller et D. Séglard, Paris, Seuil, 2000. 
Dans le moment de la décision souveraine, le peuple fait un avec celui qui, pour lui, décide. Comme par enchantement, dans la situation d'exception toutes les tensions, toutes les contradictions sont surmontées (aufgehoben?) par la décision souveraine. Dans la décision, le souverain incarne l'Un du peuple politique et doit être dit, d'après la Théorie de la constitution de I928 déjà, le vrai souverain "démocrate» dès lors que la démocratie s'entend non pas selon le schème de la représentation (la représentation déchire le corps du peuple entre représentants et représentés) mais comme identité entre Gouvernants et gouvernés. La décision souveraine du Gouvernant est le paroxysme de la démocratie schmittienne, en tant que fondée sur la décision souveraine. Dans le moment même de cette décision, le souverain apparaît comme pure objectivité, pure impartialité, car il fait alors corps avec le Peuple-Un en disant, par-delà toute "norme ", ce qu'il en est et, donc, ce qu'il doit en être du Peuple en sa forme politique. Il est donc en ce moment existentiel le plus Grand Démocrate puisque toute tension ou distance au sein même de ce peuple s'annulent dans et par la «décision».

Le coup d'État de Prusse et la participation de Schmitt à la procédure montrent in concreto ce que portent en elles la théorie du souverain et la conception de la décision, qui ne sont pas autre chose que le développement politico-juridique de la théologie politique schmittienne. Heller le met parfaitement au jour. C'est pourquoi je ne crois pas qu'on puisse faire vraiment grand-chose de la théologie politique de Schmitt, contrairement à ce que persiste à penser Jean-François Kervégan.

Mais plus encore, je ne crois pas qu'on puisse vraiment continuer de prendre au sérieux la «théorie» ou la «philosophie» du droit de Carl Schmitt. Autrement dit je veux bien admettre avec Kervégan que «nous avons besoin d'une philosophie du droit qui rende compte de la structure fine de celui-ci et des contraintes argumentatives qui sont spécifiques de son objet (par exemple: l'articulation entre droit légal et droit jurisprudentiel), sans pour autant que l'on souscrive au mythe de l'Isolierung du droit ${ }^{10}$ forgé par la

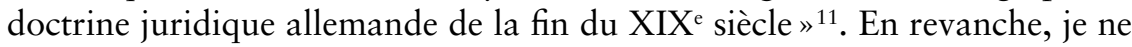
saurais souscrire à la phrase qui suit: "C'est ici que la lecture d'un auteur comme Schmitt peut être indispensable, à côté de celle de Weber ou des théoriciens institutionnalistes passés ou actuels.» Tout ce que Schmitt a à nous dire sur le droit, il l'a dit dans son seul vrai livre important sur le droit, Gesetz und Urteil (Loi et jugement) de $\mathrm{I} 9 \mathrm{I} 2^{12}$. J'argumenterai trop brièvement ici en deux temps: I) Le mythe de la décision, qu'il forge ultérieure-

10. À savoir la doctrine d'une pure autonomie de la science juridique qui pourrait se fonder elle-même, indépendamment de toute considération politique, éthique ou sociale.

11. Kervégan, op. cit., p. 75 . Sur cette doctrine allemande fin de siècle, en français: Jouanjan, Une histoire de la pensée juridique en Allemagne (1800-1918), Paris, PUF, 2005.

12. Voir, en français, l'analyse que donne Olivier Beaud de ce livre dans la préface qu'il a rédigée pour: Schmitt, Théorie de la constitution, Paris, PUF, I993. 
ment et qui n'a rien à envier, en tant que mythe, à celui de l'Isolierung, ne peut rien fonder et surtout pas une doctrine du droit; 2) le soi-disant «institutionnalisme » tardivement revendiqué par Schmitt ${ }^{13}$ n'en est pas un, et il masque mal en vérité le maintien d'une position «décisionniste " fondamentale.

I) La norme présuppose la situation normale, la situation ordonnée, et "tout ordre repose sur une décision [...] même l'ordre juridique repose, à l'instar de tout ordre sur une décision et non sur une norme ${ }^{14}$ ». C'est donc dans la situation exceptionnelle, anormale, que se révèle, comme d'elle-même, la décision en tant que fondement de tout ordre et donc aussi de l'ordre juridique. Telle est la quintessence du décisionnisme schmittien. Mais, réplique Heller, on ne peut juger de la normalité d'une situation que d'après une norme, de même qu'on ne peut juger de la positivité d'une norme que d'après sa capacité à garantir une situation (relativement) normale dans l'espace social. Et s'il faut, dans la situation "anormale" une décision, d'une part cette décision est en elle-même porteuse de norme (si elle veut avoir un effet durable et stabilisateur), d'autre part elle est justiciable d'une appréciation (juridique) au regard des normes qui déterminent ce que doit être (et non ce qu'est) une "situation normale". Il ne s'agit donc pas de nier l'importance de la décision, ni, surtout, celle de la décision dans la situation dite d'exception, mais de nier son caractère (auto)fondateur, sa qualité «souveraine» au sens de Schmitt. Car sinon, on ne comprend pas le lien, la continuité entre la décision et l'ordre, entre l'exceptionnel et le normal rétabli (ou établi) par la décision. La décision schmittienne relève du mythe et de la rhétorique et ne peut fonder aucun ordre juridique (non plus qu'aucun ordre du tout).

2) L'institutionnalisme prétendu de Schmitt, vendu sous le label «pensée de l'ordre concret", consent à reconnaître que, à la base de toute norme mais surtout de toute décision (même "souveraine») il existe un ordre "concret" qui la légitime et la justifie. Schmitt se prévaut à cet égard du grand juriste français Maurice Hauriou (I856-I929). Mais, chez ce dernier, influencé par le néo-thomisme de son époque et la philosophie de l'évolution créatrice de Bergson, l'institution est le moyen de comprendre à la fois l'unité (relative) d'une diversité fondamentale et le changement des formes de cette unité. L'institution de Hauriou doit permettre de comprendre la dialectique, dans ce que

13. Il l'est surtout au moment de l'engagement nazi de Schmitt, annoncé dans la préface à la seconde édition (1934) de la Théologie politique et développé dans ce livre qu'on ne peut décrire autrement que comme un (bref) essai de théorie nazie du droit, Les trois types de pensée juridique, paru également en 1934.

14. Schmitt, Théologie politique, trad. fr. J.-L. Schlegel, Paris, Gallimard, I988, p. 20. 
Kervégan appelle une "structure fine", entre l'un et le multiple, et entre la stabilité et le changement. L'ordre concret de Schmitt dit, au contraire, toute la pesanteur de l'Un, d'une unité mythique et mystificatrice toujours déjà là. Mais, en même temps, pour assumer l'espace de la décision dans la structure non pas fine, mais grossière de l'ordre concret, cette pensée de l'ordre s'adjoint une pensée de la Gestaltung, tout aussi «concrète". La doctrine présentée dans Les trois types de la pensée juridique de Carl Schmitt en I934 n'est donc pas une simple doctrine de l'ordre concret, mais aussi une doctrine de la pensée concrète de la Gestaltung. Ce dernier mot est intraduisible en français. Il signifie l'acte (la décision) de mise en forme plastique de la communauté politique. Chez Schmitt, cette action de mise en forme n'est pas pensée dans le cadre (pseudo-)institutionnel de l'ordre, mais comme l'intervention extérieure du souverain (en I934: du Führer). C'est pourquoi un juriste néo-hégélien, même (pour le dire brutalement) aussi nazifié que Carl Schmitt en I934, Karl Larenz, pouvait reprocher à cette pensée concrète de l'ordre et de la Gestaltung l'absence de toute médiation entre le moment du donné (l'ordre) et le moment du construit (la Gestaltung $)^{15}$. Pour le dire autrement, dans la soi-disant pensée institutionnaliste de Schmitt en I934, le décisionnisme de la Gestaltung continue de plomber la pensée de l'ordre concret, pour autant que celle-ci doive être pensée dans les termes de l'institution. L'institutionnalisme de Schmitt n'est donc qu'un pseudo-institutionnalisme qui ne parvient pas même à faire précéder la décision (Gestaltung) par l'ordre concret qui pourrait la limiter. Schmitt est resté

15. Sur tous ces points: Jouanjan, "Pensée de l'ordre concret" et ordre du discours “juridique” nazi : sur Carl Schmitt», Y. C. Zarka (dir.), Carl Schmitt ou le mythe du politique, Paris, PUF, 2009, p. 7I et sq. Kervégan cite bien ce texte, mais ne débat d'aucune façon avec lui. C'est pourquoi j'ai l'envie de relancer le débat en disant au fond que, du point de vue du juriste et malgré tout l'effort fait par Jean-François Kervégan, si rare chez les philosophes, pour lire et comprendre les juristes, son tropisme schmittien, aussi relativisé soit-il aujourd'hui, l'empêche encore de voir à quel point $S c h m i t t$, qui a eu son heure de gloire, chez les juristes allemands, jusque dans les années I980, n’est plus une référence théorique sérieuse. Je me permets une anecdote personnelle: lorsque je préparais la traduction d'un recueil d'articles d'ErnstWolfgang Böckenförde, un juriste social-démocrate proche de Carl Schmitt et ordinairement classé comme "schmittien pur et dur", nous nous sommes rencontrés, lui et moi, pour la rédaction de la présentation que j'envisageais. Je lui ai précisé que ma présentation mentionnerait certes l'influence de Schmitt, mais insisterait davantage encore sur l'importance, pour sa propre réflexion, de l'œuvre de Hermann Heller. Sa réponse, en substance et de mémoire, fut que j'avais raison et que le concept d'État comme «unité d'organisation et de décision » de Heller lui avait permis de dépasser l'aporie à laquelle conduisaient les notions d'État alors en circulation, et notamment celle de Schmitt. Je renvoie donc à ma présentation de: ErnstWolfgang Böckenförde, Le droit, l'État et la constitution démocratique. Essais de théorie juridique, politique et constitutionnelle, Paris, Bruxelles, L.G.D.J./Bruylant, coll. "La pensée juridique », 2000. 
fondamentalement décisionniste, même après son prétendu tournant « institutionnaliste».

Tout cela est bien vite dit, assurément. Carl Schmitt fascine et, par sa rhétorique exceptionnelle, embobine ses lecteurs dans la magie de son mythe. Heller est un antidote. Il montre que le concept schmittien de «peuple» n'est qu'un mythe déconnecté de la réalité sociale qui n'est pas et ne peut être "homogène ", comme le postule ou le voudrait Schmitt. La diversité et donc le conflit sont premiers, et non pas la "décision souveraine» de l'autorité incarnant l'unité homogène du "Peuple». Une saine sociologie un peu positive et réaliste permettrait de décaper la science du droit de ces scories schmittiennes. Heller ne propose pas une position "entre-deux ", un juste milieu entre le décisionnisme schmittien (même camouflé sous un pseudoinstitutionnalisme) et le normativisme kelsénien. Il nous demande de sortir de l'alternative. Pour Schmitt la décision précède toute norme et, donc, la puissance précède le droit. Pour Kelsen, la norme précède toute décision et donc la normativité juridique peut seule fonder l'État et sa puissance. Heller, lui, nous demande de penser la dialectique entre le "pouvoir créateur de puissance du droit» et le "pouvoir créateur de droit de la puissance». Il nous demande de penser dialectiquement et reproche tout autant à Schmitt qu'à Kelsen l'unilatéralité de leur pensée (que ce soit de la décision à la norme ou de la norme à la décision).

Kervégan voit parfaitement le problème Schmitt sous cet angle lorsqu'il met en évidence le type schmittien profond de pensée qui passe par un radical «ou bien... ou bien» (qui n'est évidemment pas sans rappeler le «ami/ennemi») et que, sous les auspices de Hegel, il critique l' 'unilatéralité » d'une telle pensée ${ }^{16}$. Mes regrets sont: I) que Kervégan n'ait pas suffisamment pensé avec Heller cette unilatéralité; 2) qu'il se laisse par trop emporter intellectuellement par le mythe schmittien du prétendu «peuple»; 3) qu'il se laisse tromper par le discours schmittien pseudo-institutionnaliste. Pour le reste, je le remercie d'avoir fait cet effort, si difficile et donc si remarquable, d'autocritique. Allons seulement encore un peu plus loin: il se pourrait que pour penser le droit l'on n'ait plus grand-chose à faire de Carl Schmitt. 